\title{
Article \\ Glycosylation of Ganoderic Acid G by Bacillus Glycosyltransferases
}

\author{
Jiumn-Yih Wu ${ }^{1,+}{ }^{+}$Hsiou-Yu Ding ${ }^{2,+}$, Tzi-Yuan Wang ${ }^{3,+}{ }^{\oplus}$, Yun-Rong Zhang ${ }^{4}$ and Te-Sheng Chang ${ }^{4, *} \mathbb{C}$ \\ 1 Department of Food Science, National Quemoy University, Kinmen County 892, Taiwan; wujy@nqu.edu.tw \\ 2 Department of Cosmetic Science, Chia Nan University of Pharmacy and Science, No. 60, Erh-Jen Rd., Sec. 1, \\ Jen-Te District, Tainan 71710, Taiwan; ding8896@gmail.com \\ 3 Biodiversity Research Center, Academia Sinica, Taipei 11529, Taiwan; tziyuan@gmail.com \\ 4 Department of Biological Sciences and Technology, National University of Tainan, Tainan 70005, Taiwan; \\ S10758011@gm2.nutn.edu.tw \\ * Correspondence: mozyme2001@gmail.com; Tel./Fax: +886-6-2602137 \\ + Authors contributed equally to this manuscript.
}

Citation: Wu, J.-Y.; Ding, H.-Y.; Wang, T.-Y.; Zhang, Y.-R.; Chang, T.-S. Glycosylation of Ganoderic Acid G by Bacillus Glycosyltransferases. Int. J. Mol. Sci. 2021, 22, 9744. https:// doi.org/10.3390/ijms22189744

Academic Editors:

Kateřina Valentová and

Vladimír Křen

Received: 9 August 2021

Accepted: 7 September 2021

Published: 9 September 2021

Publisher's Note: MDPI stays neutral with regard to jurisdictional claims in published maps and institutional affiliations.

Copyright: (c) 2021 by the authors. Licensee MDPI, Basel, Switzerland. This article is an open access article distributed under the terms and conditions of the Creative Commons Attribution (CC BY) license (https:// creativecommons.org/licenses/by/ $4.0 /)$.

\begin{abstract}
Ganoderma lucidum is a medicinal fungus abundant in triterpenoids, its primary bioactive components. Although numerous Ganoderma triterpenoids have already been identified, rare Ganoderma triterpenoid saponins were recently discovered. To create novel Ganoderma saponins, ganoderic acid G (GAG) was selected for biotransformation using four Bacillus glycosyltransferases (GTs) including BtGT_16345 from the Bacillus thuringiensis GA A07 strain and three GTs (BsGT110, BsUGT398, and BsUGT489) from the Bacillus subtilis ATCC 6633 strain. The results showed that BsUGT489 catalyzed the glycosylation of GAG to GAG-3-O- $\beta$-glucoside, while BsGT110 catalyzed the glycosylation of GAG to GAG-26-O- $\beta$-glucoside, which showed 54 -fold and 97 -fold greater aqueous solubility than that of GAG, respectively. To our knowledge, these two GAG saponins are new compounds. The glycosylation specificity of the four Bacillus GTs highlights the possibility of novel Ganoderma triterpenoid saponin production in the future.
\end{abstract}

Keywords: Bacillus; Ganoderma lucidum; glycosyltransferase; saponin; triterpenoid

\section{Introduction}

Ganoderma lucidum is a medicinal fungus that has been used for health improvement and the treatment of various diseases in Asia over thousands of years [1]. In modern studies, both triterpenoids and polysaccharides have been demonstrated to exhibit bioactivity [2]. Triterpenoids contain six isoprene units and usually form tetra- or penta-cyclic structures. Triterpenoids are widely noted to exist in plants and some fungi. Until now, more than 14,000 different triterpenoids have been identified, and many of them have been proven to play important roles in bioactivity [3]. Triterpenoid saponins are important natural products in plants, and some triterpenoid saponins exhibit greater bioactivity than triterpenoid aglycones [4]. Therefore, the glycosylation of triterpenoids is important for the biosynthesis of triterpenoid saponins. G. lucidum also contains large amounts of triterpenoids, and over 300 triterpenoids that form Ganoderma sp. have been identified previously [5-7]. All Ganoderma triterpenoids belong to the tetracyclic lanostane type of triterpenoids which contain different types of main skeletons, including ganoderic acids. These Ganoderma triterpenoids were found to possess varying degrees of bioactivity. For example, a Ganoderma triterpenoid, ganoderic acid A (GAA), was recently shown to have bioactive medical applications including the inhibition of bleomycin-induced lung fibrosis in mice [8], the attenuation of lipopolysaccharide-induced lung injury in mice [9], the retardation of renal cyst development in polycystic kidney disease [10], and the protection of neural cells against NO stress injury [11]. Considering the diversity of the Ganoderma triterpenoid group, studying the bioactivity of Ganoderma triterpenoids has become a core 
focus of biotechnology studies in recent years. Consequently, rare Ganoderma triterpenoid saponins have been identified recently [5-7].

In nature, the glycosylation of triterpenoids is initiated by glycosyltransferase (EC 2.4.1.X; GT) [12]. GT uses a nucleotide-activated sugar donor, such as uridine diphosphate glucose (UDP-G), to transfer the sugar moiety to a sugar acceptor. According to the carbohydrate-activating enzyme database there are 117 subfamilies of GTs, with over 850,000 GTs having already been identified [13]. However, only some rare bacterial GTs are able to glycosylate triterpenoids to triterpenoid saponins [14]. In our previous studies, we used GAA as a precursor to screen microbes with the ability to biotransform GAA to GAA saponins. Two bacteria, the Bacillus subtilis (Bs) American Type Culture Collection (ATCC) 6633 strain and the Bacillus thuringiensis (Bt) GA A07 strain, were able to produce GAA saponins. Four Bacillus GTs were further identified, based on their genomes, as the major enzymes able to catalyze the glycosylation of GAA to GAA saponins. BsGT110 [15], BsUGT398, and BsUGT489 [16] were identified using the ATCC 6633 strain, and BtGT_16345 was identified using the GA A07 strain [17]. So far, only seven Bacillus GTs with triterpenoid glycosylation activity have been identified, including the four Bacillus GTs mentioned previously [14]. Phylogenetic analysis suggests that the four Bacillus GTs are genetically distinct from each other (Figure 1) [14]. Although the four Bacillus GTs have been proven to have the ability to produce Ganoderma triterpenoid saponins, previous studies have only focused on GAA glycosylation [15-17]. Thus, it is worth investigating the catalytic activity of the four Bacillus GTs toward other triterpenoids.

Therefore, the glycosylation sites of ganoderic acid G (GAG), namely, the C-3, C-7, and $\mathrm{C}-12$ hydroxyl groups and the C-26 carboxyl group, were selected to test the biotransformation of the four Bacillus GTs. The glycosylated saponins produced by the four Bacillus GTs using GAG were purified and their chemical structures were identified. In addition, the aqueous solubility of the GAG saponins was determined and compared with that of GAG. Accordingly, the catalytic specificity of the four Bacillus GTs toward the different functional groups of the ganoderic acids was clarified.

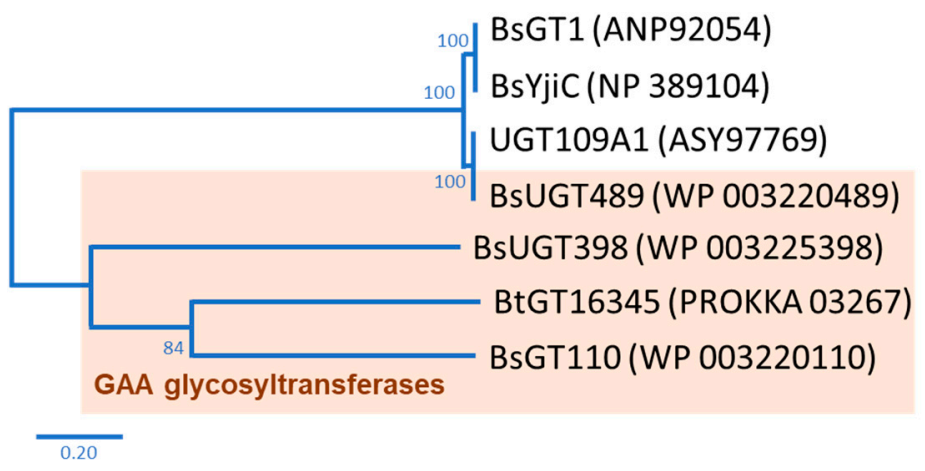

Figure 1. Seven known bacterial glycosyltransferases (GTs) from previous studies. The phylogenetic tree was adopted from Figure 1 in the study by Chang et al. [14].

\section{Results and Discussion}

\subsection{Biotransformation}

Ganoderic acid is one of most common types of Ganoderma triterpenoids and primarily contains five functional groups that are available for modification, including the C-3, C-7, C-12, and C-15 hydroxyl groups and the C-26 carboxyl group [5-7]. In this study, the four Bacillus GTs (Figure 1) were assayed for their glycosylation activity toward GAG containing C-3, C-7, and C-12 hydroxyl groups and the C-26 carboxyl group (Figure 2). After the completion of the biotransformation reaction, the reaction mixture was analyzed using high-performance liquid chromatography (HPLC), the results of which are shown in Figure 3. The results indicate that BsUGT489 and BsGT110 biotransformed GAG to compound (1) and compound (2), with yields of $74 \%$ and $35 \%$, respectively. Both BsUGT398 and BtGT_16345 showed little to no activity toward GAG, respectively. 


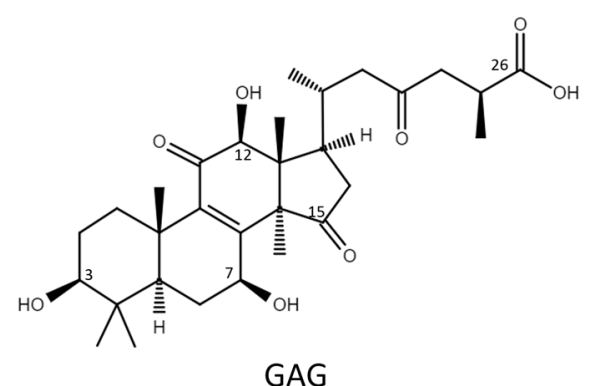

Figure 2. The chemical structure of ganoderic acid G (GAG).

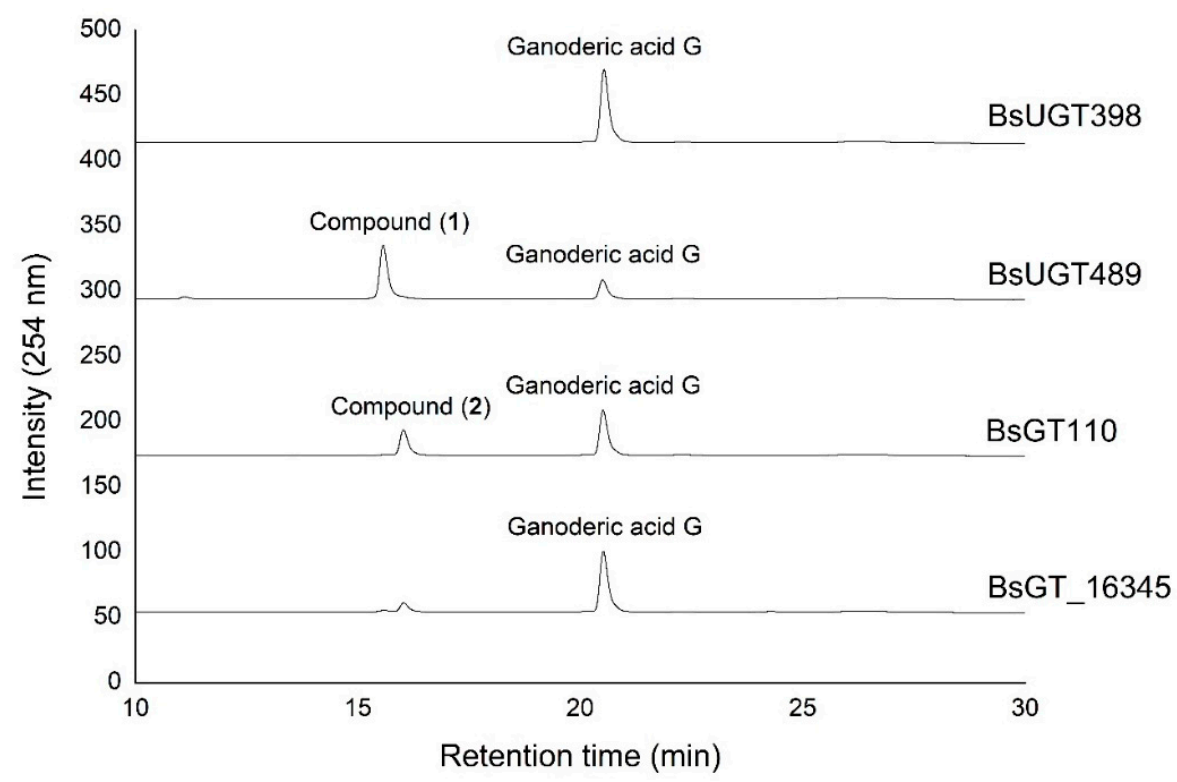

Figure 3. High-performance liquid chromatography (HPLC) results of the biotransformation products of GAG using the four Bacillus GT enzymes. The biotransformation and HPLC conditions are described in the Materials and Methods section.

\subsection{Purification and Identification of Biotransformed Products}

The biotransformation reactions of GAG with BsUGT489 and BsGT110 were selected to purify compound (1) and compound (2), respectively. The two biotransformation reactions were scaled up to $20 \mathrm{~mL}$ and the products were purified by preparative HPLC. From the $20 \mathrm{~mL}$ reaction mixture, $14.4 \mathrm{mg}$ of compound (1) and $7.8 \mathrm{mg}$ of compound (2) were purified. The molecular weights of the purified products were then determined by mass spectrometry. The mass spectrometer showed an $[\mathrm{M}-\mathrm{H}]^{-}$ion peak at $\mathrm{m} / \mathrm{z}: 693.5$ in the electrospray ionization mass spectrum (ESI-MS), corresponding to the molecular formula $\mathrm{C}_{36} \mathrm{H}_{54} \mathrm{O}_{13}$. The mass data imply that both compound (1) and compound (2) contain one glucosyl moiety attached to the GAG structure (Figures S1 and S2). To identify the structures in advance, the structures of both products were determined using nuclear magnetic resonance (NMR) spectroscopy. The ${ }^{1} \mathrm{H}$ and ${ }^{13} \mathrm{C} N \mathrm{NR}$, including the distortionless enhancement by polarization transfer (DEPT), heteronuclear multiple bond connectivity (HMBC), heteronuclear single quantum coherence (HSQC), nuclear Overhauser effect spectroscopy (NOESY), and correlation spectroscopy (COSY) spectra were obtained. The NMR spectra of compound (1) exhibited characteristic glucosyl signals, with the anomeric proton signal at $\delta_{\mathrm{H}} 3.98\left({ }^{1} \mathrm{H}, \mathrm{ddd}, J=8.6,5.6,2.1 \mathrm{~Hz}, \mathrm{H}-5^{\prime}\right), 4.03\left({ }^{1} \mathrm{H}, \mathrm{t}\right.$, $\left.J=8.6 \mathrm{~Hz}, \mathrm{H}-2^{\prime}\right), 4.22\left({ }^{1} \mathrm{H}, \mathrm{t}, J=8.6 \mathrm{~Hz}, \mathrm{H}-4^{\prime}\right), 4.24\left({ }^{1} \mathrm{H}, \mathrm{t}, J=8.6 \mathrm{~Hz}, \mathrm{H}-3^{\prime}\right), 4.40\left({ }^{1} \mathrm{H}, \mathrm{dd}\right.$, $\left.J=11.9,5.6 \mathrm{~Hz}, \mathrm{H}-6^{\prime} \mathrm{a}\right), 4.57\left({ }^{1} \mathrm{H}, \mathrm{dd}, J=11.9,2.1 \mathrm{~Hz}, \mathrm{H}-6^{\prime} \mathrm{b}\right)$, and $4.91\left({ }^{1} \mathrm{H}, \mathrm{d}, J=8.6 \mathrm{~Hz}\right.$, $\left.\mathrm{H}-1^{\prime}\right)$; and the anomeric carbon signal at $\delta_{\mathrm{C}} 63.0\left(\mathrm{C}-6^{\prime}\right), 71.8\left(\mathrm{C}-4^{\prime}\right), 75.7\left(\mathrm{C}-2^{\prime}\right), 78.4\left(\mathrm{C}-5^{\prime}\right)$, $78.7\left(\mathrm{C}-3^{\prime}\right)$, and $107.0\left(\mathrm{C}-1^{\prime}\right)$. The large coupling constant $(8.6 \mathrm{~Hz})$ of the anomeric proton 
$\mathrm{H}-1^{\prime}$ (4.91 ppm) indicated the $\beta$-configuration. An ether linkage between the $\mathrm{H}-\mathrm{1}^{\prime}$ of glucose and C-3 (4.91/88.3 ppm) of GAG was proven by the HMBC and NOESY (H-3/H-1 $\left.{ }^{\prime}\right)$ spectra. The structure of compound (1) was thus confirmed to be GAG-3-O- $\beta$-glucoside. The NMR spectroscopic data are shown in Figures S3-S9. The signals of compound (2) were attributed to a glucose moiety, with $\delta_{\mathrm{H}} 4.03\left({ }^{1} \mathrm{H}, \mathrm{ddd}, J=8.8,4.9,2.8 \mathrm{~Hz}, \mathrm{H}-5^{\prime}\right), 4.20$ $\left({ }^{1} \mathrm{H}, \mathrm{t}, J=8.8 \mathrm{~Hz}, \mathrm{H}-2^{\prime}\right), 4.29\left({ }^{1} \mathrm{H}, \mathrm{t}, J=8.8 \mathrm{~Hz}, \mathrm{H}-3^{\prime}\right), 4.35\left(1 \mathrm{H}, \mathrm{t}, J=8.8 \mathrm{~Hz}, \mathrm{H}-4^{\prime}\right), 4.36\left({ }^{1} \mathrm{H}\right.$, $\left.\mathrm{dd}, J=11.9,4.9 \mathrm{~Hz}, \mathrm{H}-6^{\prime} \mathrm{a}\right), 4.46\left({ }^{1} \mathrm{H}, \mathrm{dd}, J=11.9,2.8 \mathrm{~Hz}, \mathrm{H}-6^{\prime} \mathrm{b}\right)$, and $6.33\left({ }^{1} \mathrm{H}, \mathrm{d}, J=8.8 \mathrm{~Hz}\right.$, $\left.\mathrm{H}-1^{\prime}\right)$; and $\delta_{\mathrm{C}} 62.1\left(\mathrm{C}-6^{\prime}\right), 71.0\left(\mathrm{C}-4^{\prime}\right), 74.2\left(\mathrm{C}-2^{\prime}\right), 78.5\left(\mathrm{C}-3^{\prime}\right), 79.5\left(\mathrm{C}-5^{\prime}\right)$, and $96.3\left(\mathrm{C}-1^{\prime}\right)$. The cross peak of $\mathrm{H}-1^{\prime}$ with $\mathrm{C}-26(6.33 / 175.0 \mathrm{ppm})$ in the $\mathrm{HMBC}$ spectrum demonstrated the structure of compound (2) to be GAG-26-O- $\beta$-glucoside. The NMR spectroscopic data are shown in Figures S10-S16. The structures of the GAG saponins and the biotransformation process are shown in Figure 4.

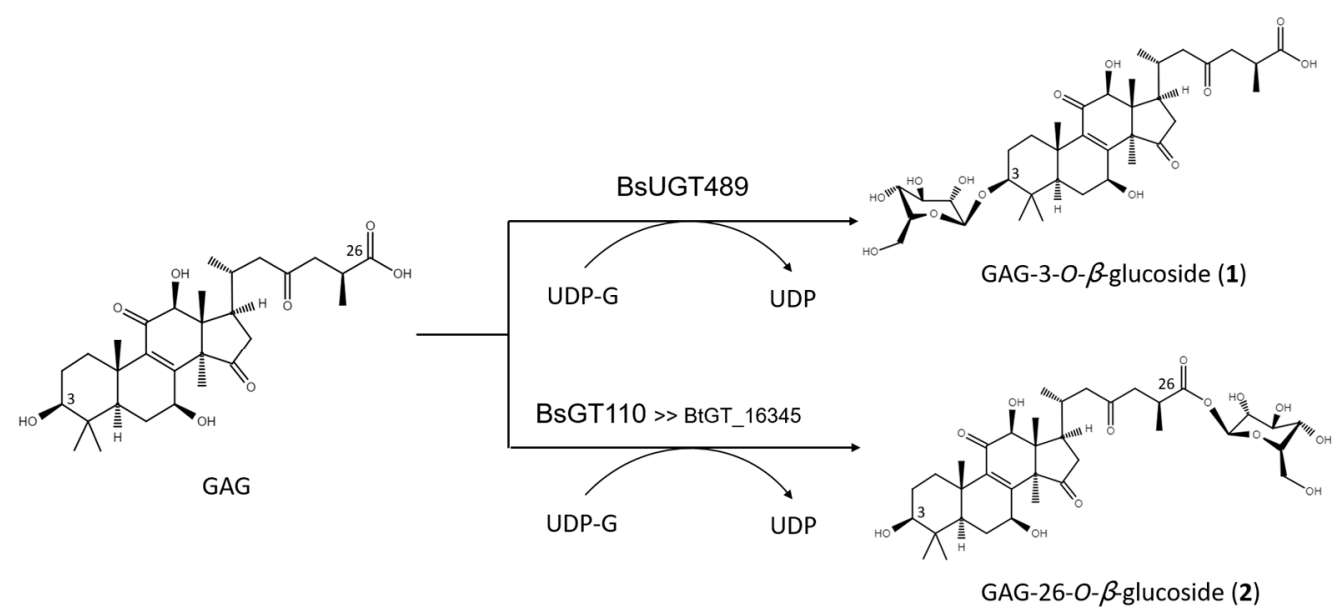

Figure 4. The biotransformation process of GAG to GAG saponins by the Bacillus GTs.

2.3. Catalytic Specificity of the Bacillus GTs for the Glycosylation of Functional Groups on Ganoderic Acids

The four Bacillus GTs have been considered genetically distinct among the known triterpenoid-catalyzed GTs until now (Figure 1). Previous studies revealed that BsUGT398, BsUGT489, and BtGT_16345 have catalytic specificity toward glycosylating the C-15 hydroxyl group of the GAA triterpenoid [16,17]. In addition, BsGT110 was the first GT that glycosylated the C-26 carboxyl group of the GAA triterpenoid. Moreover, BsGT110 has shown optimal activity at an acidic $\mathrm{pH}$ of 6, but a loss of most activity at an alkaline $\mathrm{pH}$ of 8. This suggests that BsGT110 might catalyze other triterpenoid substrates under acidic conditions [15], and is further confirmed by our results. BsGT110 also glycosylated the carboxyl group of GAG under acidic condition (Figures 3 and 4). In addition, BsUGT489 was found to glycosylate the C-3 hydroxyl group of GAG. Table 1 and Figure 5 summarize the comparative results of the biotransformation of GAA [15-17] and GAG with the four Bacillus GTs. The main structure of ganoderic acid contains five functional groups that could be modified: the C-3, C-7, C-12, and C-15 hydroxyl groups, and the C-26 carboxyl group. GAA and GAG are ganoderic acid derivatives. The structure of GAA contains the C-7 and C-15 hydroxyl groups, and the C-26 carboxyl group. The structure of GAG contains the C-3, C-7, and C-12 hydroxyl groups and the C-26 carboxyl group. Accordingly, previous results revealed that (1) BsGT110 regioselectively glycosylates the C-26 carboxyl groups of both GAA and GAG; (2) both BtGT_16345 and BsUGT398 prefer to glycosylate the C-15 hydroxyl group of GAA; (3) BsUGT489 glycosylates the C-3 and C-15 hydroxyl groups of GAG and GAA, respectively. In none of these cases did the four Bacillus GTs glycosylate the C-7 or C-12 hydroxyl groups of GAA or GAG. We theorize that the enzymatic preferences of these Bacillus GTs may enable the formation of new Ganoderma triterpenoid saponins in the future. 
Table 1. Glycosylation activities of the four Bacillus glycosyltransferases (GTs) toward the different functional groups of GAA and GAG.

\begin{tabular}{cccccc}
\hline \multirow{2}{*}{ Triterpenoid } & \multicolumn{5}{c}{ Functional Groups in Ganoderic Acids } \\
\cline { 2 - 6 } GAA & C3-OH & C7-OH & C12-OH & C15-OH & C26-COOH \\
\hline GAG & N.A. ${ }^{1}$ & N.D. ${ }^{2}$ & N.A. & BsUGT489 > BtGT_16345> \\
BsUGT398
\end{tabular}

${ }^{1}$ N.A. means that the functional group is not available in the ganoderic acid. ${ }^{2}$ N.D. means that the glycosylation of the functional group was not detectable in the reactions of the studied GTs. ${ }^{3}$ Data obtained from Table 1 of Ref. [17].

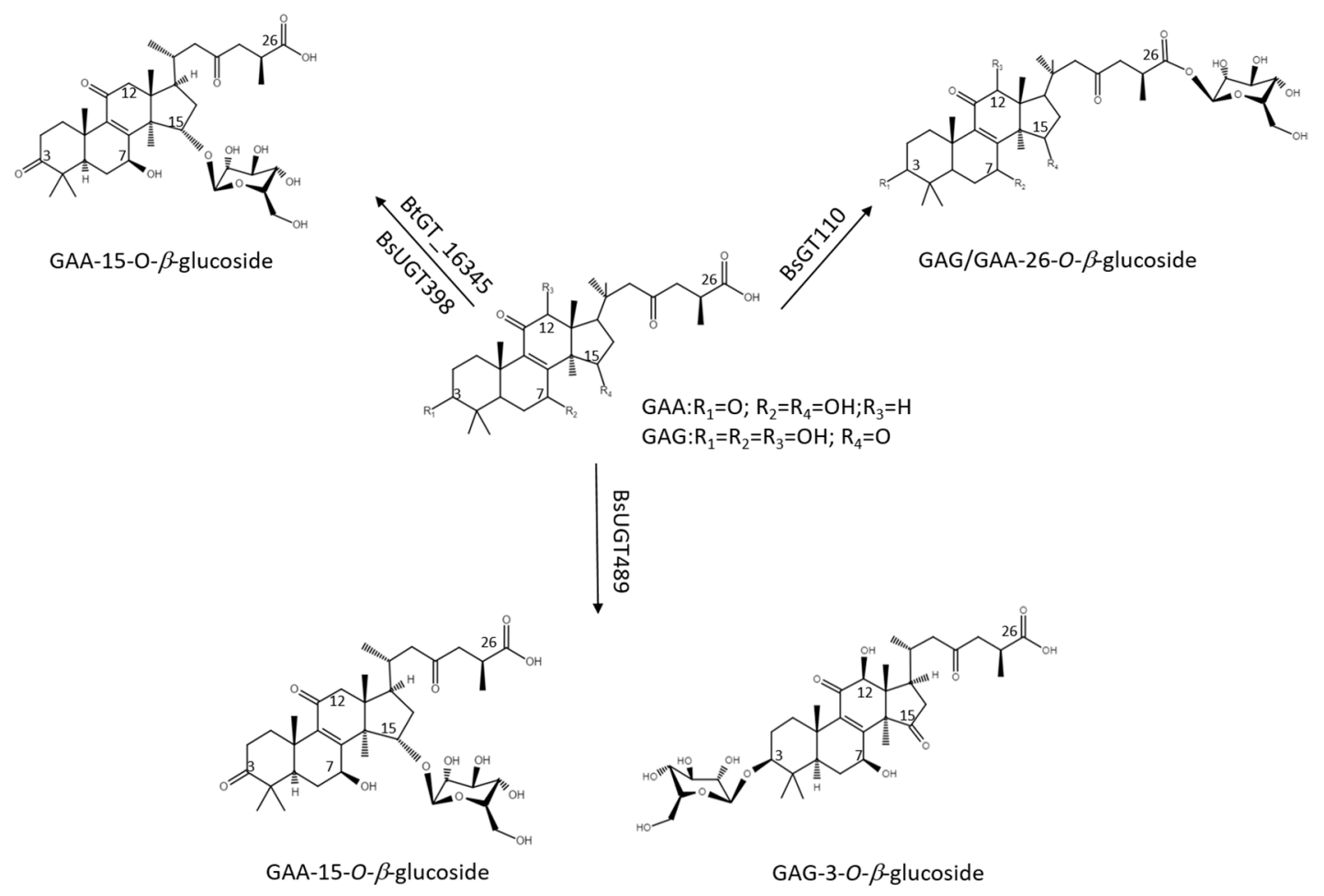

Figure 5. The glycosylation of GAA and GAG by the four Bacillus GTs.

\subsection{Determination of Solubility}

The glycosylated molecules demonstrated more advantages than the original aglycone molecules. After glycosylation, the aqueous solubility of the glycosylated derivatives was greatly increased [18,19]. Accordingly, the aqueous solubility of GAG and the two GAG saponins are listed in Table 2. These results show that the aqueous solubility of compound (1) and compound (2) were 54- and 97-fold greater than that of GAG. Previous studies revealed that glycosylation can also improve the oral bioavailability of the original molecules [20,21]. Thus, the pharmaceutical application of both novel GAG saponins might be worthy of future study.

Table 2. Aqueous solubility of GAG and its derivatives at $25^{\circ} \mathrm{C}$.

\begin{tabular}{ccc}
\hline Compound & Aqueous Solubility (mg/L) $^{\mathbf{1}}$ & Fold $^{\mathbf{2}}$ \\
\hline GAG & $18.85 \pm 1.36$ & 1 \\
GAG-3-O- $\beta$-glucoside (1) & $1019.64 \pm 9.54$ & 54.0 \\
GAG-26-O- $\beta$-glucoside (2) & $1829.89 \pm 51.21$ & 97.0 \\
\hline
\end{tabular}

${ }^{1}$ The mean $(n=3)$ are shown, and the standard deviations are represented by error bars. ${ }^{2}$ The fold of aqueous solubility of GAG glucoside derivatives is expressed as relative to that of GAG, normalized to 1. 


\subsection{Perspective}

The biological reason for the joint involvement of GAG and Bacillus GTs in the synthesis of fungi saponins remains unclear. However, most of the prior studies about amphiphilic molecules have sought to determine, in advance, those saponins capable of inducing cell toxicity, and we know from these studies that some saponins possess membrane permeabilizing properties. Moreover, saponins have been shown to cause mortality, lower food intake, weight reduction, and developmental problems in insects [22,23]. Plants contain large amounts of triterpenoid saponins and saponins, in turn, avoid plants infected by insect bites. On the other hand, glycosylation is also a well-known detoxification process during drug metabolism [24]. It has been reported that triterpenoids are toxic to bacteria (possessing anti-bacterial activity) and that the glycosylation of triterpenoids to saponins reduces triterpenoids toxic effects on bacteria. Accordingly, one possible explanation is that GTs help bacteria to survive with the fungi by glycosylating the fungal triterpenoids to detoxed saponins.

\section{Materials and Methods}

\subsection{Enzymes and Chemicals}

GAG was purchased from Baoji Herbest Bio-Tech (Xi'an, China). UDP-G was obtained from Cayman Chemical (Ann Arbor, MI, USA). The recombinant Bacillus GTs, BsGT110 [15], BsUGT398, BsUGT489 [16], and BtGT_16345 [17] were obtained from our previous studies. Organic solvents, including methanol and dimethyl sulfoxide (DMSO), and nylon membrane were bought from Sigma (St. Louis, MO, USA).

\subsection{Biotransformation}

The in vitro biotransformation was conducted using the purified recombinant GTs. The optimal reaction conditions for GAA glycosylation, suggested in previous studies, were used as the reaction conditions for GAG glycosylation [15-17]. The reaction mixture ( $1 \mathrm{~mL}$ ) contained $25 \mu \mathrm{g} / \mathrm{mL}$ of the tested enzyme, $1 \mathrm{mg} / \mathrm{mL}$ of GAG, $10 \mathrm{mM}$ of UDP-G, $10 \mathrm{mM}$ of $\mathrm{MgCl}_{2}$, and $50 \mathrm{mM}$ of buffer at different $\mathrm{pHs}$ (see below). The reactions were carried out at $30{ }^{\circ} \mathrm{C}$ for BsUGT398 and BtGT_16345 for $30 \mathrm{~min}$, and at $40{ }^{\circ} \mathrm{C}$ for BsUGT489 and BsGT110 for $30 \mathrm{~min}$. For different enzyme reactions, different buffers were used: phosphate buffer (PB) at pH 6.0 for BsGT110, PB at pH 7.0 for BtGT_16345, and Tris buffer at pH 8.0 for BsUGT398 and BsUGT489. Reactions were ended by adding an equal volume of methanol and the mixture was then analyzed using HPLC.

\subsection{HPLC Analysis}

HPLC was performed using the Agilent ${ }^{\circledR} 1100$ series HPLC system (Santa Clara, CA, USA) equipped with a gradient pump (Waters 600, Waters, Milford, MA, USA). The stationary phase was a C18 column (Sharpsil H-C18, $5 \mu \mathrm{m}, 4.6$ i.d. $\times 250 \mathrm{~mm}$, Sharpsil, Bei-jing, China) and the mobile phase was $1 \%$ acetic acid in water (A) and methanol (B). The elution condition was a linear gradient from 0 min with $40 \%$ B to 20 min with $70 \%$ $\mathrm{B}$; isocratic from $20 \mathrm{~min}$ to $25 \mathrm{~min}$ with $70 \% \mathrm{~B}$; linear gradient from $25 \mathrm{~min}$ with $70 \% \mathrm{~B}$ to 28 min with $40 \% \mathrm{~B}$; and isocratic from $28 \mathrm{~min}$ to $35 \mathrm{~min}$ with $40 \% \mathrm{~B}$. The flow rate of the mobile phase was $1 \mathrm{~mL} / \mathrm{min}$. The sample volume was $10 \mu \mathrm{L}$. Detection intensity was set at $254 \mathrm{~nm}$.

\subsection{Purification and Identification of the Biotransformation Products}

To purify the biotransformation metabolites, the biotransformation reactions using either BsUGT489 or BtGT_16345, described above, were scaled up to $20 \mathrm{~mL}$. Equal volumes of methanol were added to stop the reactions. After filtration through a $0.2 \mu \mathrm{m}$ nylon membrane, the mixtures were injected into a preparative YoungLin HPLC system (YL9100, YL Instrument, Anyang, Korea). The system was equipped with a preparative C18 reversedphase column (Inertsil, $10 \mu \mathrm{m}, 20.0$ i.d. $\times 250 \mathrm{~mm}$, ODS 3, GL Sciences, Eindhoven, The Netherlands). The operational conditions for the preparative HPLC were the same 
as those for HPLC. The elution corresponding to the peak of the product in UPLC was collected, concentrated under vacuum, and then lyophilized. Finally, $14.4 \mathrm{mg}$ of compound (1) and $7.8 \mathrm{mg}$ of compound (2) were obtained, and the structures of both compounds were confirmed using NMR and mass spectrometry. Mass spectrometry was performed using a Finnigan LCQ Duo mass spectrometer (ThermoQuest Corp., San Jose, CA, USA) with ESI. The ${ }^{1} \mathrm{H}$ - and ${ }^{13} \mathrm{C}-\mathrm{NMR}$, DEPT, HSQC, HMBC, COSY, and NOESY spectra were recorded using a Bruker AV-700 NMR spectrometer (Bruker Corp., Billerica, MA, USA) at $25^{\circ} \mathrm{C}$. Standard pulse sequences and parameters were used for the NMR analyses and all chemical shifts were reported in parts per million (ppm, $\delta$ ).

\subsection{Determination of Solubility}

The aqueous solubility of GAG and its glucoside derivatives were examined as follows. One milligram of each compound was vortexed in $0.1 \mathrm{~mL}$ of deionized water for $1 \mathrm{~h}$ at $25^{\circ} \mathrm{C}$. The mixture was then centrifuged at $10,000 \times g$ for $30 \mathrm{~min}$ and the supernatant was filtered through a $0.2 \mu \mathrm{m}$ nylon membrane at $25^{\circ} \mathrm{C}$. The filtrate was mixed with an equal volume of methanol and analyzed using HPLC. The concentrations of the tested compounds were determined based on their peaks using calibration curves prepared using HPLC analyses of the authentic samples dissolved in DMSO.

\section{Conclusions}

Two new and highly aqueous-soluble Ganoderma triterpenoid saponins, GAG-3-O$\beta$-glucoside (1) and GAG-26-O- $\beta$-glucoside (2), were produced by the biotransformation of GAG using BsUGT489 and BsGT110, respectively. This study revealed that the glycosylation site preferences of the different functional ganoderic acid groups vary among the different Bacillus GTs. BsGT110 regioselectively glycosylates the C-26 carboxyl group of GAA and GAG. Both BtGT_16345 and BsUGT398 prefer to glycosylate the C-15 hydroxyl group of GAA. BsUGT489 glycosylates the C-3 and C-15 hydroxyl groups of GAG and GAA, respectively. This study highlights the possible application of Bacillus GTs for purposes of forming future Ganoderma triterpenoid saponins.

Supplementary Materials: The following materials are available online at https:/ www.mdpi.com/ article/10.3390/ijms22189744/s1.

Author Contributions: Conceptualization: T.-S.C.; data curation and methodology: Y.-R.Z., T.-S.C. and H.-Y.D.; project administration: T.-S.C. and J.-Y.W.; writing-original draft, review, and editing: T.-S.C., T.-Y.W., J.-Y.W. and H.-Y.D. All authors have read and agreed to the published version of the manuscript.

Funding: This research was funded by the Ministry of Science and Technology of Taiwan, grant number MOST 110-2221-E-024-002 to T.-S.C. and grant number MOST 110-2221-E-507-002 to J.-Y.W.

Institutional Review Board Statement: Not applicable.

Informed Consent Statement: Not applicable.

Data Availability Statement: The data presented in this study are available in the article or supplementary material.

Conflicts of Interest: The authors declare no conflict of interest.

\section{References}

1. Ahmad, M.F. Ganoderma lucidum: Persuasive biologically active constituents and their health endorsement. Biomed. Pharmacother. 2018, 107, 507-519. [CrossRef]

2. Xu, J.W.; Zhao, W.; Zhong, J.J. Biotechnological production and application of ganoderic acids. Appl. Microbiol. Biotechnol. 2010, 87, 457-466. [CrossRef]

3. Sultana, N.; Saify, Z.S. Enzymatic biotransformation of terpenes as bioactive agents. J. Enzym. Inhib. Med. Chem. 2013, 28, 1113-1128. [CrossRef]

4. Shi, Z.Y.; Zeng, J.Z.; Wong, A.S.T. Chemical structures and pharmacological profiles of ginseng saponins. Molecules 2019, $24,2443$. [CrossRef] [PubMed] 
5. Yang, Y.; Zhang, H.; Zuo, J.; Gong, X.; Yi, F.; Zhu, W.; Li, L. Advances in research on the active constituents and physiological effects of Ganoderma lucidum. Biomed. Dermatol. 2019, 3, 6. [CrossRef]

6. Liang, C.Y.; Tian, D.N.; Liu, Y.Z.; Li, H.; Zhu, J.L.; Li, M.; Xin, M.H.; Xia, J. Review of the molecular mechanisms of Ganoderma lucidum triterpenoids: Ganoderic acids A, C2, D, F, DM, X and Y. Eur. J. Med. Chem. 2019, 174, 130-141. [CrossRef] [PubMed]

7. Xia, Q.; Zhang, H.Z.; Sun, X.F.; Zhao, H.J.; Wu, L.F.; Zhu, D.; Yang, G.H.; Shao, Y.Y.; Zhang, X.X.; Mao, X.; et al. A comprehensive review of the structure elucidation and biological activity of triterpenoids from Ganoderma spp. Molecules 2014, 19, 17478-17535. [CrossRef] [PubMed]

8. Wen, G.; Li, T.; He, H.; Zhou, X.; Zhu, J. Ganoderic Acid A Inhibits Bleomycin-Induced Lung Fibrosis in Mice. Pharmacology 2020, 105, 568-575. [CrossRef] [PubMed]

9. Wan, B.; Li, Y.; Sun, S.; Yang, Y.; Lv, Y.; Wang, L.; Song, M.; Chen, M.; Wu, C.; Pan, H.; et al. Ganoderic acid A attenuates lipopolysaccharide-induced lung injury in mice. Biosci. Rep. 2019, 39, BSR20190301. [CrossRef] [PubMed]

10. Meng, J.; Wang, S.Z.; He, J.Z.; Zhu, S.Z.; Huang, B.Y.; Wang, S.Y.; Li, M.; Zhou, H.; Lin, S.Q.; Yang, B.X. Ganoderic acid A is the effective ingredient of Ganoderma triterpenes in retarding renal cyst development in polycystic kidney disease. Acta Pharmacol. Sin. 2020, 41, 782-790. [CrossRef]

11. Yu, Z.R.; Jia, W.H.; Liu, C.; Wang, H.Q.; Yang, H.G.; He, G.R.; Chen, R.Y.; Du, G.H. Ganoderic acid A protects neural cells against NO stress injury in vitro via stimulating $\beta$ adrenergic receptors. Acta Pharmacol. Sin. 2020, 41, 516-522. [CrossRef]

12. Mestrom, L.; Przypis, M.; Kowalczykiewicz, D.; Pollender, A.; Kumpf, A.; Marsden, S.R.; Bento, I.; Jarzebski, A.B.; Szymanska, K.; Chrusciel, A.; et al. Leloir glycosyltransferases in applied biocatalysis: A multidisciplinary approach. Int. J. Mol. Sci. 2019, 20, 5263. [CrossRef] [PubMed]

13. Cantarel, B.L.; Coutinho, P.M.; Rancurel, C.; Bernard, T.; Lombard, V.; Henrissat, B. The Carbohydrate-Active EnZymes database (CAZy): An expert resource for Glycogenomics. Nucleic Acids Res. 2009, 37, D233-D238. [CrossRef]

14. Chang, T.S.; Wang, T.Y.; Chiang, C.M.; Lin, Y.J.; Chen, H.L.; Wu, Y.W.; Ting, H.J.; Wu, J.Y. Biotransformation of celastrol to a novel, well-soluble, low-toxic and anti-oxidative celastrol-29-O-beta-glucoside by Bacillus glycosyltransferases. J. Biosci. Bioeng. 2021, 131, 176-182. [CrossRef]

15. Chang, T.S.; Chiang, C.M.; Kao, Y.H.; Wu, J.Y.; Wu, Y.W.; Wang, T.Y. A new triterpenoid glucoside from a novel acidic glycosylation of ganoderic acid A via recombinant glycosyltransferase of Bacillus subtilis. Molecules 2019, 24, 3457. [CrossRef] [PubMed]

16. Chang, T.S.; Wu, J.Y.; Wang, T.Y.; Wu, K.Y.; Chiang, C.M. Uridine diphosphate-dependent glycosyltransferases from Bacillus subtilis ATCC 6633 catalyze the 15-O-glycosylation of ganoderic acid A. Int. J. Mol. Sci. 2018, 19, 3469. [CrossRef]

17. Chang, T.S.; Wang, T.Y.; Hsueh, T.Y.; Lee, Y.W.; Chuang, H.M.; Cai, W.X.; Wu, J.Y.; Chiang, C.M.; Wu, Y.W. A genome-centric approach reveals a novel glycosyltransferase from the GA A07 Strain of Bacillus thuringiensis responsible for catalyzing 15-Oglycosylation of ganoderic acid A. Int. J. Mol. Sci. 2019, 20, 5192. [CrossRef] [PubMed]

18. Moulis, C.; Guieysse, D.; Morel, S.; Severac, E.; Remaud-Simeon, M. Natural and engineered transglycosylases: Green tools for the enzyme-based synthesis of glycoproducts. Curr. Opin. Chem. Biol. 2021, 61, 96-106. [CrossRef]

19. Slamova, K.; Kapesova, J.; Valentova, K. “Sweet Flavonoids”: Glycosidase-catalyzedmodifications. Int. J. Mol. Sci. 2018, 19, 2126. [CrossRef]

20. Zhao, J.; Yang, J.; Xie, Y. Improvement strategies for the oral bioavailability of poorly water-soluble flavonoids: An overview. Int. J. Pharm. 2019, 570, 118642. [CrossRef] [PubMed]

21. Fu, J.; Wu, Z.; Zhang, L. Clinical applications of the naturally occurring or synthetic glycosylated low molecular weight drugs. Progress Mol. Biol. Transl. Sci. 2019, 163, 487-522. [CrossRef]

22. Agerbirk, N.; Olsen, C.E.; Bibby, B.M.; Frandsen, H.O.; Brown, L.D.; Nielsen, J.K.; Renwick, J.A. A saponin correlated with variable resistance of Barbarea vulgaris to the diamondback moth Plutella xylostella. J. Chem. Ecol. 2003, 29, 1417-1433. [CrossRef]

23. Christensen, S.; Enge, S.; Jensen, K.R.; Müller, C.; Kiær, L.P.; Agerbirk, N.; Heimes, C.; Hauser, T.P. Different herbivore responses to two co-occurring chemotypes of the wild crucifer Barbarea vulgaris. Arthropod Plant Interact. 2019, 13, 19-30. [CrossRef]

24. Poppenberger, B.; Berthiller, F.; Lucyshyn, D.; Sieberer, T.; Schuhmacher, R.; Krska, R.; Kuchler, K.; Glossl, J.; Luschnig, C.; Adam, G. Detoxification of the Fusarium mycotoxin deoxynivalenol by a UDP-glucosyltransferase from Arabidopsis thaliana. $J$. Biol. Chem. 2003, 278, 47905-47914. [CrossRef] [PubMed] 